\title{
Minimal Edit-Based Diffs for Large Trees
}

\author{
Mateusz Pawlik \\ University of Salzburg \\ Salzburg, Austria \\ mateusz.pawlik@sbg.ac.at
}

\author{
Nikolaus Augsten \\ University of Salzburg \\ Salzburg, Austria \\ nikolaus.augsten@sbg.ac.at
}

\begin{abstract}
Hierarchically structured data are commonly represented as trees and have given rise to popular data formats like XML or JSON. An interesting query computes the difference between two versions of a tree, expressed as the minimum set of node edits (deletion, insertion, label rename) that transform one tree into another, commonly known as the tree edit distance. Unfortunately, the fastest tree edit distance algorithms run in cubic time and quadratic space and are therefore not feasible for large inputs. In this paper, we leverage the fact that the difference between two versions of a tree is typically much smaller than the overall tree size. We propose a new tree edit distance algorithm that is linear in the tree size for similar trees. Our algorithm is based on the new concept of top node pairs and avoids redundant distance computations, the main issue with previous solutions for tree diffs. We empirically evaluate the runtime of our algorithm on large synthetic and real-world trees; our algorithm clearly outperforms the state of the art, often by orders of magnitude.
\end{abstract}

\section{CCS CONCEPTS}

- Information systems $\rightarrow$ Query operators; Deduplication; Data cleaning.

\section{KEYWORDS}

tree edit distance; minimal tree difference; tree-structured data; similarity search

\section{ACM Reference Format:}

Mateusz Pawlik and Nikolaus Augsten. 2020. Minimal Edit-Based Diffs for Large Trees. In Proceedings of the 29th ACM International Conference on Information and Knowledge Management (CIKM '20), October 19-23, 2020, Virtual Event, Ireland. ACM, New York, NY, USA, 10 pages. https://doi.org/ $10.1145 / 3340531.3412026$

\section{INTRODUCTION}

The tree edit distance (TED) expresses the difference between two trees as the minimum number of node edits (deletion, insertion, and label change) that transform one tree into another. Thanks to its minimality guarantee and its intuitive interpretation, TED has been successfully applied in a wide range of applications, for example, in information extraction [9,17], document retrieval [15], similarity queries [3, 11, 27], image analysis [6], pattern recognition [18],

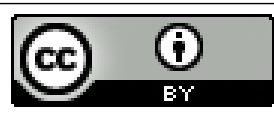

This work is licensed under a Creative Commons Attribution International 4.0 License.

CIKM '20, October 19-23, 2020, Virtual Event, Ireland

(C) 2020 Copyright held by the owner/author(s).

ACM ISBN 978-1-4503-6859-9/20/10.

https://doi.org/10.1145/3340531.3412026 melody recognition [12], natural language processing [19], and bioinformatics $[1,2,13,20]$.

The goal of this work is to efficiently compute the tree edit distance between two versions of very large data trees with hundreds of thousands of nodes. Unfortunately, the fastest TED algorithms $[21,23]$ run in cubic time and quadratic space, and faster solutions are unlikely to exist [8]. General TED algorithms clearly do not scale to large trees. We observe, however, that these solutions do not take advantage of the similarity of the input trees, i.e., the runtime for identical trees is the same as for very different trees. This is unfavorable for diff computations since the difference between two tree versions is often much smaller than the tree size.

In this paper, we present TopDiff, a new algorithm that leverages the similarity of two tree versions to scale the diff computation to large trees. Our algorithm is inspired by the method of Touzet [25]. Touzet uses a dynamic programming approach and builds the solution for larger instances from the solutions of smaller subproblems (a subproblem is a pair of subtrees of the input trees). Hütter et al.[14] show how to use Touzet's algorithm to compute the edit distance if an upper bound $\tau$ on the distance is known. Given the upper bound $\tau$, this algorithm runs in $O\left(n \tau^{3}\right)$ time and $O(n \tau)$ space for trees with $n$ nodes.

Touzet's algorithm uses a number of pruning techniques that leverage the upper bound $\tau$ to achieve linear runtime in the tree size. Unfortunately, these pruning techniques come at a price: they are not compatible with well-known techniques to avoid redundant computations of subproblems in dynamic programming algorithms for TED, e.g., keyroot nodes by Zhang and Shasha [29] or top light nodes by Demaine et al. [10]. Therefore, Touzet's algorithm may compute a large number of subproblems multiple times. As we show in our experimental evaluation, these redundant computations often lead to slow runtimes in practice.

Our TopDiff algorithm solves the problem of redundant computations. We introduce the concept of top node pairs, which mark the subproblems in the two input trees that must be considered. This is challenging since the relevant subproblems depend on the upper bound $\tau$ and on the structure of both input trees, i.e., they cannot be precomputed on each input tree in isolation (like keyroot nodes [29]). Top node pairs guarantee that no subproblem is computed more than once, and we develop an efficient algorithm to compute all top node pairs in linear time.

Let the depth $d$ be the maximum length of a root-leaf path in a tree. TopDiff runs in linear space and time $O\left(n d \tau^{2}\right)$, i.e., the algorithm is linear for shallow trees with constant depth $d$. Most real-world tree data sets enjoy this property, e.g., Barbosa et al. [5] found an average depth of 4 and a maximum depth of 135 in a sample of 200,000 XML documents on the web. To avoid the quadratic worst case for deep trees, we design the TopDiff ${ }^{+}$algorithm which uses top node pairs for flat trees and applies depth-based 
pruning [25] for deep trees. TopDiff ${ }^{+}$achieves $O\left(n \tau^{3}\right)$ runtime (like Touzet's algorithm), but at the same time avoids redundant computations for shallow trees with depth $d=O(\tau)$. We propose a structure-aware cost function to switch between the techniques and empirically evaluate its accuracy. We show that, depending on the tree structure, it may be beneficial to use top node pairs also for deep trees.

We analyze the complexity of TopDiff, TopDiff ${ }^{+}$, and Touzet's algorithm and provide fine-grained complexity bounds that consider the tree structure for all algorithms. We show that our algorithms enjoy tighter bounds for some tree shapes.

In our tree diff scenario, no upper bound $\tau$ on the distance is known. Upper bounds for the tree edit distance have been proposed, but they are either too slow (e.g., the constrained edit distance runs in quadratic time [28]) or too loose for large trees (e.g., the label guided mapping [14] overestimates the distance between the tree versions in our experiments by more than two orders of magnitude). Since the runtime is cubic in the upper bound $\tau$, overestimating this parameter will adversely affect the runtime. Our approach is to start with a lower bound on the distance and incrementally double the bound until it is large enough. We prove the correctness of this technique, which increases the overall complexity only by a logarithmic factor in the upper bound $\tau$.

Summarizing, we make the following contributions:

- We introduce the TopDiff algorithm that uses the novel top node pairs technique to solve the problem of redundant distance computations found in the state-of-the-art solution.

- We design the TopDiff ${ }^{+}$algorithm and use it to solve the edit-based tree diff problem in $O\left(n \delta^{3} \log \delta\right)$ time, where $\delta$ is the size of the minimal diff. TopDiff ${ }^{+}$avoids redundant computations for shallow trees with depth $d=O(\delta)$ and enables depth-based pruning using on a novel cost formula.

- We analyze the runtime complexity of TopDiff ${ }^{+}$and the state of the art for tree diffs and prove new structure-aware complexity bounds.

- Our experimental evaluation confirms that our approach scales to tree instances with hundreds of thousands of nodes and outperforms the state of the art in both the number of computed subproblems and the overall runtime, often by orders of magnitude.

The paper has the following structure. Section 2 reviews the related works. We introduce necessary definitions in Section 3. Section 4 revisits the algorithm by Touzet. We introduce TopDiff in Section 5. We explain how to deal with deep trees and propose TopDiff $^{+}$in Section 6. Section 7 discusses how to compute TED independently on the upper bound value. We evaluate the algorithms experimentally in Section 8 and conclude in Section 9.

\section{RELATED WORK}

Exact Tree Edit Distance. The tree edit distance was proposed by Tai [24] in 1979 with an $O\left(n^{6}\right)$-time solution, where $n$ is the input tree size. Since then, a number of works improved the time complexity. The current state-of-the-art solution is APTED by Pawlik and Augsten [23] that runs in $O\left(n^{3}\right)$ time and $O\left(n^{2}\right)$ space. Bringmann et al. [8] prove that a truly subcubic-time algorithm for TED is

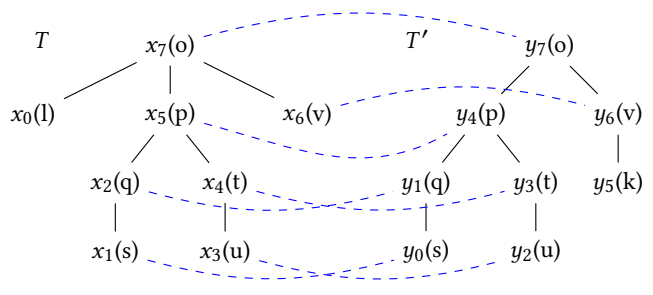

Figure 1: Running example tree pair.

unlikely to exist. Cubic runtime and quadratic space are not feasible for large trees that we target in this work.

Touzet [25] assumes a known upper bound $\tau$ for the distance between two trees and designs an algorithm with $O\left(n \tau^{3}\right)$-time and $O(n \tau)$-space bounds. To the best of our knowledge, this is the only algorithm for TED that can deal with large trees. Touzet devises pruning techniques to reduce the search space of potential solutions. However, her techniques introduce redundant computations that lead to high runtimes, especially for flat trees. Our TopDiff algorithm is efficient for flat trees and is guaranteed to avoid redundancies. We empirically compare our solution to that of Touzet.

Upper bounds. Both the algorithm by Touzet [25] and our solution, TopDiff, require an upper bound to compute TED. Unfortunately, the available upper bounds are either inefficient or inaccurate. The most recent survey of different upper bounds is by Kan et al. [16]. All upper bound algorithms reviewed and proposed in [16] require $O\left(n^{2}\right)$ time and are therefore infeasible for large trees. The constrained tree edit distance by Zhang [28] is the only upper bound that requires less than quadratic, $O(n \log n)$, space [26].

A linear-time upper bound algorithm has recently been proposed by Hütter et al. [14] in the scenario of similarity joins. The so-called Label Guided Mapping (LGM) executes in $O(n \tau)$ time and requires linear space. The algorithm greedily maps nodes with identical labels and adds additional node mappings while observing the edit mapping conditions. It prunes any node pairs that would lead to a mapping with a cost higher than a given $\tau$. The accuracy of LGM primarily depends on the number of different labels. The algorithm chooses the first node pair with identical labels that can be mapped according to $\tau$. If this pair is not part of the optimal mapping, the error may quickly propagate leading to high distance values, especially for large trees. For example, for tree pair L3 (cf. Section 8) and $\tau=113$ (exact TED value) the LGM upper bound value is 80438 .

We develop an iterative solution that uses our TopDiff ${ }^{+}$algorithm and computes TED without a prior knowledge on the upper bound in $O\left(n \delta^{3} \log \delta\right)$ time, where $\delta$ is the edit distance between the input trees.

Approximations. Approximation algorithms for TED have received little attention. Recently, Boroujeni et al. [7] developed a $(1+\epsilon)$-approximation for TED. The algorithm requires $O\left(n^{2}\right)$ time. The authors review also other approximation techniques for TED.

\section{PRELIMINARIES}

We recap basic definitions related to TED algorithms [14] and summarize the most important notations in Table 1. 
Table 1: List of notations

\begin{tabular}{ll}
\hline Notation & Description \\
\hline$T, T^{\prime}$ & two trees \\
$\operatorname{root}(T)$ & root node of tree $T$ \\
$x_{i} \in T$ & node $x$ in tree $T$ with postorder ID $i$ \\
post $(x)$ & postorder ID of node $x$ \\
$\operatorname{depth}(x)$ & depth of node $x$; depth $(\operatorname{root}(T))=0$ \\
$\operatorname{depth}(T)$ & depth of tree $T ; \max _{x} \in T\{\operatorname{depth}(x)\}$ \\
$\delta\left(T, T^{\prime}\right)$ & tree edit distance between $T$ and $T^{\prime}$ \\
$\operatorname{cost}(M)$ & cost of an edit mapping $M$ \\
$a_{x}, d_{x}, l_{x}, r_{x}$ & number of $x$ 's ancestors, descendants, \\
& nodes to the left, nodes to the right \\
$v_{x}=\left(l_{x}, d_{x}, a_{x}, r_{x}\right)$ & neighborhood vector of node $x$ \\
$\left\|v_{x}-v_{y}\right\|_{1}$ & neighborhood distance of $x$ and $y$ \\
$\tau$ & upper bound value on TED \\
$|p o s t(x)-p o s t(y)|$ & postorder difference \\
$\epsilon(x, y, \tau)$ & edits budget for mapping $T_{x}$ and $T_{y}^{\prime}$ \\
$l l d(x)$ & leftmost leaf descendant of node $x$ \\
$>_{l}$ & partial order between node pairs \\
\hline
\end{tabular}

Trees. A tree $T$ is a directed, acyclic, connected graph with nodes $N(T)$ and edges $E(T) \subseteq N(T) \times N(T)$, where each node has at most one incoming edge. We abbreviate $x \in N(T)$ with $x \in T$. The size $|T|$ of tree $T$ is the number of its nodes. In an edge $(x, y), x$ is the parent and $y$ is the child, $p(y)=x$; children of the same node are siblings. Node $x$ is an ancestor of node $y$ iff $x=p(y)$ or $x$ is an ancestor of $p(y) ; x$ is a descendant of $y$ iff $y$ is an ancestor of $x$. The depth of a node $x$, depth $(x)$, is the number of $x$ 's ancestors. The depth of a tree $T$ is $\operatorname{depth}(T)=\max _{x \in T}\{\operatorname{depth}(x)\}$. We denote a subtree of tree $T$ rooted at node $x$ as $T_{x} ; T_{x}$ consists of node $x$, all its descendants, and all edges between these nodes. The trees are rooted (the node with no incoming edge is the root node, root $(T)$ ) and ordered (the sibling order matters). Each node $x$ has a label, that carries the data of the node. Labels are not necessarily unique. The postorder id of node $x$, post $(x)$, is its postorder position in the tree (zero-based numbering) that is assigned to the node by a postorder traversal of a tree. A postorder traversal visits children before parents, and siblings in order. A formal definition of postorder can be found in [23]. In our examples, we identify the nodes with their postorder IDs written as a subscript of a node. $x$ is to the left of $y$ if post $(x)<\operatorname{post}(y)$ and $x$ is not a descendant of $y$; $x$ is to the right of $y$ if post $(x)>\operatorname{post}(y)$ and $x$ is not an ancestor of $y$.

Example 3.1. Figure 1 introduces trees $T$ and $T^{\prime}$, which we use as a running example. The subscript of a node is its postorder ID, the label is shown in braces. Node $x_{7}$ is the root of tree $T, x_{0}, x_{1}, x_{3}, x_{6}$ are leaves. Node $x_{4}$ has one descendant $\left(x_{3}\right)$, two ancestors $\left(x_{5}, x_{7}\right)$, three nodes to the left $\left(x_{0}, x_{1}, x_{2}\right)$, one node to the right $\left(x_{6}\right)$.

Tree Edit Distance (TED). The edit distance, $\delta\left(T, T^{\prime}\right)$, is the minimum number of node edit operations that transform tree $T$ into tree $T^{\prime}[22,29]$. The node edit operations are: delete node $x$ and connect all its children to the parent of $x$ maintaining the sibling order; insert a new node $y$ between an existing node $x$ and a consecutive subsequence of $x$ 's children, rename the label of node $x$.

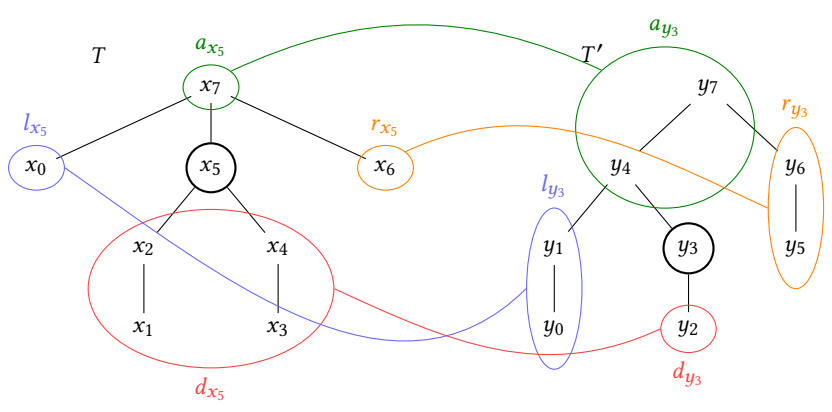

Figure 2: Neighborhood distance example.

Edit Mapping. An edit mapping, $M$, between the nodes of trees $T$ and $T^{\prime}$, represents the edit operations that transform $T$ into $T^{\prime}$. Node pairs that are mapped are renamed, unmapped nodes in $T$ are deleted, unmapped nodes in $T^{\prime}$ are inserted. Nodes with identical labels are renamed at zero cost, all other operations have cost 1.

Definition 3.2. Edit mapping. A mapping $M \subseteq T \times T^{\prime}$ is an edit mapping from $T$ to $T^{\prime}$ if for any two node pairs $(x, y),\left(x^{\prime}, y^{\prime}\right) \in M$ the following conditions hold:

- $x=x^{\prime}$ iff $y=y^{\prime}$ (one-to-one),

- $x$ is an ancestor of $x^{\prime}$ iff $y$ is an ancestor of $y^{\prime}$ (ancestor),

- $x$ is to the left of $x^{\prime}$ iff $y$ is to the left of $y^{\prime}$ (order).

The cost of an edit mapping, $\operatorname{cost}(M)$, is the cost sum of all its operations. The cost of the optimal edit mapping (mapping with minimum cost) between $T$ and $T^{\prime}$ is their edit distance [29].

Example 3.3. In Figure 1, the optimal mapping $M$ between trees $T$ and $T^{\prime}$ is shown with blue dashed lines. All mapped nodes correspond to renames of cost 0 ; node $x_{0}$ is deleted, node $y_{5}$ is inserted, both at $\operatorname{cost} 1: \operatorname{cost}(M)=\delta\left(T, T^{\prime}\right)=2$.

\section{TOUZET'S ALGORITHM REVISITED}

Neighborhood distance. Given a mapped node pair $(x, y) \in M$, the edit mapping conditions impose restrictions on further mappings in $M$. These can occur only between specific partitions of the neighborhoods of $x$ and $y$, i.e., ancestors, descendants, nodes to the left, and nodes to the right (see color-coded mapped regions in Figure 2). These restrictions can be leveraged to compute a cost lower bound on the mapping given a single mapped node pair [14, 25]. Let $a_{x}, d_{x}, l_{x}, r_{x}$ denote the number of ancestors, descendants, nodes to the left, and nodes to the right of node $x$, respectively. We call $v_{x}=\left(l_{x}, d_{x}, a_{x}, r_{x}\right)$ the neighborhood vector of node $x$. The neighborhood vector is computed in constant time from postorder ID, node depth, and subtree size of node $x: l_{x}=\operatorname{post}(x)-\left|T_{x}\right|+1$, $d_{x}=\left|T_{x}\right|-1, a_{x}=\operatorname{depth}(x), r_{x}=|T|-\operatorname{post}(x)-\operatorname{depth}(x)-1$.

Definition 4.1. Neighborhood distance. The neighborhood distance, $\left\|v_{x}-v_{y}\right\|_{1}$ of nodes $x$ and $y$ is the $L_{1}$ distance between their neighborhood vectors:

$$
\left\|v_{x}-v_{y}\right\|_{1}=\left|l_{x}-l_{y}\right|+\left|d_{x}-d_{y}\right|+\left|a_{x}-a_{y}\right|+\left|r_{x}-r_{y}\right|
$$

Given an upper bound value $\tau$ on TED, such that $\delta\left(T, T^{\prime}\right) \leq \tau$, the neighborhood distance can be used to prune allowable edit 


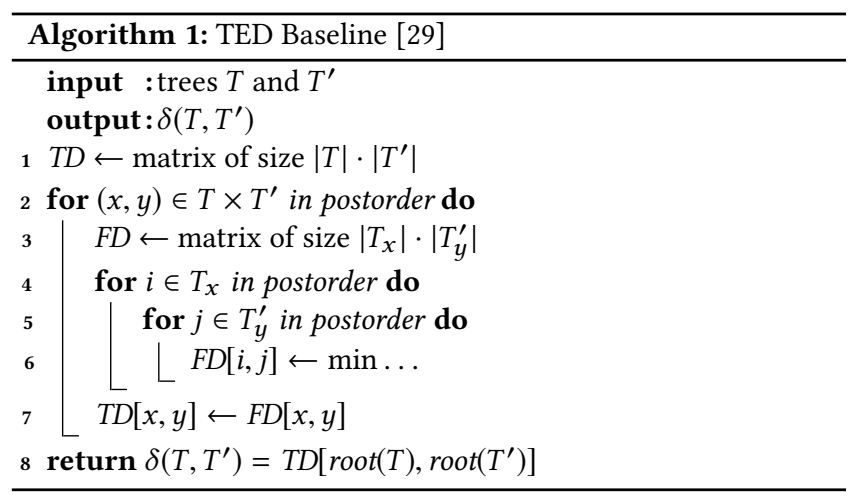

operations with the following lemma. Intuitively, if a node pair is mapped, its neighborhood distance is a lower bound on the number of deletions and insertions induced by such a pair.

LEMma 4.2. [25] The neighborhood distance of any node pair $(x, y)$ in a mapping with $\operatorname{cost}(M) \leq \tau$ is no larger than $\tau$.

$$
\operatorname{cost}(M) \leq \tau \Rightarrow \forall_{(x, y) \in M}\left\|v_{x}-v_{y}\right\|_{1} \leq \tau
$$

A mapping $M$ between $T$ and $T^{\prime}$ with $\operatorname{cost}(M) \leq \tau$ exists iff the edit distance $\delta\left(T, T^{\prime}\right) \leq \tau$. For a pair $(x, y) \in M$, $\operatorname{cost}(M) \leq \tau$, we draw the following conclusions. (1) The postorder difference of the mapped nodes is no larger than $\tau$, $|\operatorname{post}(x)-\operatorname{post}(y)| \leq \tau$, due to $\operatorname{post}(x)=l_{x}+d_{x}$. (2) The edits budget, $\epsilon(x, y, \tau)$, defined as the maximum number of edits that transform the subtree $T_{x}$ into $T_{y}^{\prime}$ in mapping $M$, is limited to $\epsilon(x, y, \tau)=\tau-\left|l_{x}-l_{y}\right|-\left|a_{x}-a_{y}\right|-\left|r_{x}-r_{y}\right|$.

Example 4.3. Consider Figure 2. We check if the node pair $\left(x_{5}, y_{3}\right)$ (circled in black) can be in a mapping $M$ with $\operatorname{cost}(M) \leq \tau=2$. The postorder difference, $\left|\operatorname{post}\left(x_{5}\right)-\operatorname{post}\left(y_{3}\right)\right|=2$, is no larger than $\tau$, i.e., the pair passes this filter. The figure shows how the neighborhoods of $x_{5}\left(l_{x_{5}}\right.$ : nodes to the left, $d_{x_{5}}$ : descendants, $a_{x_{5}}$ : ancestors, $r_{x_{5}}$ : nodes to the right) are mapped to the respective neighborhoods of $y_{3}$. The neighborhood distance, $\left\|v_{x_{5}}-v_{y_{3}}\right\|_{1}=6$, is larger than $\tau$, i.e., the pair $\left(x_{5}, y_{3}\right)$ cannot be in $M$. An example of a pair that passes the neighborhood filter for $\tau=2$ is $\left(x_{4}, y_{3}\right)$ with $\left\|v_{x_{4}}-v_{y_{3}}\right\|_{1}=2$. The edits budget for $\left(x_{4}, y_{3}\right)$ is $\epsilon\left(x_{4}, y_{3}, 2\right)=$ $2-\left|l_{x_{4}}-l_{y_{3}}\right|+\left|a_{x_{4}}-a_{y_{3}}\right|+\left|r_{x_{4}}-r_{y_{3}}\right|=0$, i.e., if $\left(x_{4}, y_{3}\right) \in M$, $\operatorname{cost}(M) \leq 2$, then $M$ must transform $T_{x}$ to $T_{y}^{\prime}$ at zero cost.

Touzet [25] extends the TED baseline by Zhang and Shasha [29] with three pruning techniques that leverage the upper bound $\tau$, which is assumed to be given: (1) subtree pruning, (2) edits pruning, and (3) depth-based pruning. We revisit the TED baseline, discuss subtree and edits pruning, and defer depth-based pruning to Sect. 6 .

TED Baseline. For the input trees $T$ and $T^{\prime}$, the baseline Algorithm 1 computes the distances between all subtree pairs $\left(T_{x}, T_{y}^{\prime}\right)$ using dynamic programming. The subtree pairs are traversed in increasing postorder of their root nodes (line 2), and the results for larger subtree pairs are computed from smaller subproblems. The distance values are stored in matrix $T D$, the subproblems that must be computed for a given subtree pair $\left(T_{x}, T_{y}^{\prime}\right)$ are stored in a temporary matrix $F D$ (lines 3-6). A subproblem for a node pair $(i, j) \in T_{x} \times T_{y}^{\prime}$ in $F D$ is computed in line 6 as the minimum over a
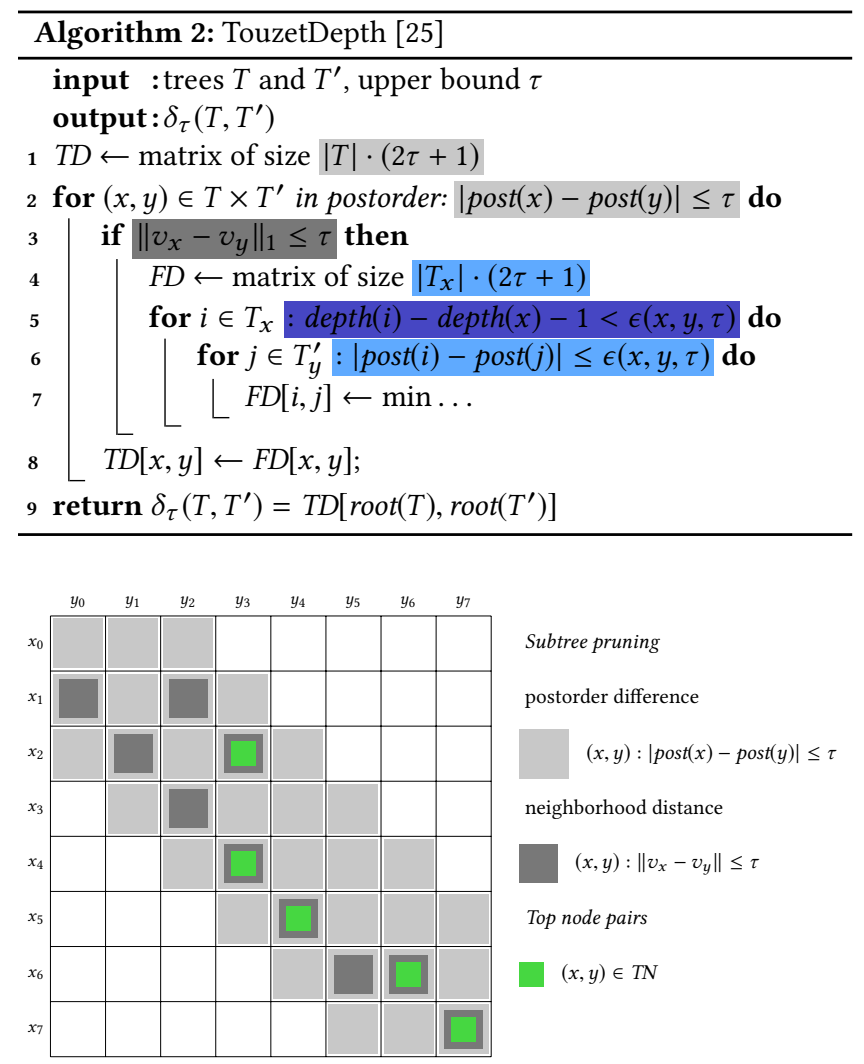

Figure 3: Example matrix $T D$ and subtree pruning for $\tau=2$.

constant number of values already stored in TD and FD. More details and a correctness proof can be found in Zhang and Shasha [29].

The baseline algorithm runs in $O\left(n^{4}\right)$ time and $O\left(n^{2}\right)$ space. Runtime: The number of subtree pairs $\left(T_{x}, T_{y}^{\prime}\right)$ is $|T| \cdot\left|T^{\prime}\right|$; each node pair $(i, j) \in T_{x} \times T_{y}^{\prime}$ is processed in constant time; the subtree sizes $T_{x}\left(T_{y}^{\prime}\right)$ are bound by $\left|T_{x}\right| \leq|T|\left(\left|T_{y}^{\prime}\right| \leq\left|T^{\prime}\right|\right)$. Space: Matrices $T D$ and $F D$ dominate the memory; their size is bound by $|T| \cdot\left|T^{\prime}\right|$.

Subtree Pruning. Algorithm 2 shows Touzet's extension of the baseline (Algorithm 1). Subtree pruning reduces the number of node pairs $(x, y)$ for which the subtree distance must be computed, i.e., the number of cells in matrix TD that are filled: If $\left\|v_{x}-v_{y}\right\|_{1}>\tau$, the node pair $(x, y)$ is not in the optimal mapping (cf. Lemma 4.2), thus subtree pair $\left(T_{x}, T_{y}^{\prime}\right)$ can be pruned. Subtree pruning is implemented as follows: The outer loop in line 2 considers node pairs that are eligible based on their postorder difference as introduced in Section 3 (light gray). In line 3, the neighborhood distance is checked (dark gray), which is stricter than the postorder difference.

Example 4.4. Figure 3 shows matrix TD for our example trees in Figure 1 . A cell in row $x_{i}$ and column $y_{j}$ stores the distance between subtrees $T_{x_{i}}$ and $T_{y_{j}}$. The baseline algorithm computes all cells. The cells that pass the postorder difference condition are marked in light gray, the cells that also pass the stricter neighborhood distance condition are marked in dark gray. In total, subtree pruning reduces the number of subtree computations from 64 to 10 . 


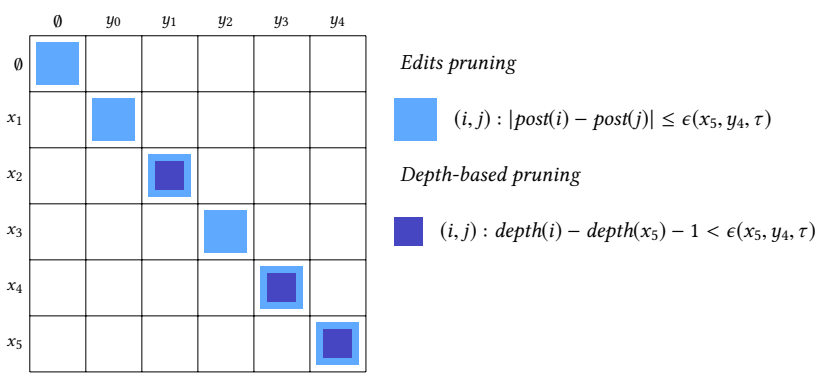

Figure 4: Matrix $\boldsymbol{F D}$, edits pruning for subtrees $T_{x_{5}}, T_{y_{4}}^{\prime}, \tau=2$.

Edits pruning. The distance between a subtree pair $\left(T_{x}, T_{y}^{\prime}\right)$ is computed in a nested loop and stored in matrix FD (lines 5-7, Algorithm 2). The baseline must fill all cells of $F D$, i.e., $\left|T_{x}\right| \cdot\left|T_{y}^{\prime}\right|$ values. Edits pruning leverages the edits budget, $\epsilon(x, y, \tau)$, which limits the number of edits that are allowed for a given subtree pair (cf. Section 3). Each row (column) in FD refers to a node $i \in T_{x}$ $\left(j \in T_{y}^{\prime}\right)$ and marks a subproblem ( $\emptyset$ is the empty subproblem). Since only $\epsilon(x, y, \tau)$ edits are allowed, all pairs with postorder difference $|\operatorname{post}(i)-\operatorname{post}(j)|>\epsilon(x, y, \tau)$ can be pruned (light blue, line 6).

Example 4.5. Figure 4 shows matrix $F D$ for the example subtrees $T_{x_{5}}$ and $T_{y_{4}}^{\prime}$ in Figure 1. For upper bound $\tau=2$, the edits budget $\epsilon\left(x_{5}, y_{4}, \tau\right)=0$, i.e., no edits are allowed for this subtree pair. Only the diagonal values, for which $|\operatorname{post}(i)-\operatorname{post}(j)| \leq 0$, need to be computed, thus reducing the number of subproblems from 36 to 6 .

Complexity analysis. Subtree pruning: For a node $x \in T$, at most $2 \tau+1$ nodes satisfy the postorder condition (line 2, Algorithm 2), i.e., for each row in $T D$ at most $2 \tau+1$ column values are computed. Edits pruning: For each node $i \in T_{x}$ at most $2 \epsilon\left(x_{i}, x_{j}, \tau\right)+1$ nodes $j \in T_{y}^{\prime}$ satisfy the condition $|\operatorname{post}(i)-\operatorname{post}(j)| \leq \epsilon\left(x_{i}, x_{j}, \tau\right)$. Since $\epsilon\left(x_{i}, x_{j}, \tau\right) \leq \tau,\left|T_{x}\right| \leq|T|$, at most $|T| \cdot(2 \tau+1)$ values are computed in FD. Overall, the runtime complexity is $O\left(n^{2} \tau^{2}\right)$ for trees of size $n$ and the memory complexity is $O(n \tau)$ [25].

\section{REMOVING REDUNDANT COMPUTATIONS}

The baseline and Touzet's algorithm (cf. Section 4) may compute a single subproblem multiple times: Two different node pairs $(x, y)$ in the outer loop (Algorithm 2, line 2) may require the computation of identical subproblems in the inner loops (lines 5-7).

This is a well-known problem that has been addressed by Zhang and Shasha [30] for the baseline algorithm by introducing so-called keyroot nodes. All nodes except the first child of each parent are keyroot nodes. Only node pairs $(x, y)$ such that both $x$ and $y$ are keyroots must be considered in the outer loop. For example, the keyroot nodes of tree $T$ and $T^{\prime}$ in Figure 1 are $x_{4}, x_{5}, x_{6}, x_{7}$ and $y_{3}, y_{6}, y_{7}$, respectively.

When a keyroot node is considered, all nodes on the leftmost path to a leaf (i.e., the path that always follows the edge to the first child) are computed as a by-product. For example, when keyroot pair $\left(x_{5}, y_{7}\right)$ is considered, the distances between all pairs $\left\{x_{1}, x_{2}, x_{5}\right\} \times\left\{y_{0}, y_{1}, y_{4}, y_{7}\right\}$ are computed, and these distances will not be computed for another keyroot pair.

Unfortunately, keyroots are not compatible with the pruning techniques of Algorithm 2 by Touzet. As an example, consider the keyroot pair $\left(x_{5}, y_{7}\right)$ and $\tau=2$. Due to the neighborhood distance $\left\|x_{5}-y_{7}\right\|_{1}=6$ this pair is pruned. Therefore, none of the pairs $\left\{x_{1}, x_{2}, x_{5}\right\} \times\left\{y_{0}, y_{1}, y_{4}, y_{7}\right\}$ will be considered, and these pairs cannot appear in the mapping. However, the pairs $\left(x_{1}, y_{0}\right),\left(x_{2}, y_{1}\right)$, and $\left(x_{5}, y_{4}\right)$ are in the optimal mapping, i.e., pruning the keyroot pair $\left(x_{5}, y_{7}\right)$ leads to an incorrect result.

Top node pairs. We introduce the concept of top node pairs to avoid redundant computations. We define the leftmost leaf descendant, $\operatorname{lld}(x)$, of node $x$, as the node in subtree $T_{x}$ with the smallest postorder ID. Intuitively, the leftmost leaf descendant is reached from $x$ by always following the edge to the first child. The postorder ID of the leftmost leaf descendant is post $(\operatorname{lld}(x))=\operatorname{post}(x)-\left|T_{x}\right|+1$.

We further define a partial order, $>_{l}$, between node pairs $(x, y)$, $\left(x^{*}, y^{*}\right) \in T \times T^{\prime}$ based on common leftmost leaf descendants: $\left(x^{*}, y^{*}\right)>_{l}(x, y) \Leftrightarrow \operatorname{post}\left(x^{*}\right) \geq \operatorname{post}(x) \wedge \operatorname{post}\left(y^{*}\right) \geq \operatorname{post}(y) \wedge$ $\operatorname{lld}\left(x^{*}\right)=\operatorname{lld}(x) \wedge \operatorname{lld}\left(y^{*}\right)=\operatorname{lld}(y) \wedge\left(x \neq x^{*} \vee y \neq y^{*}\right)$. For example, $\left(x_{2}, y_{4}\right)>_{l}\left(x_{2}, y_{1}\right)$ in Figure 1 since post $\left(y_{4}\right) \geq \operatorname{post}\left(y_{1}\right)$, and $\operatorname{lld}\left(y_{4}\right)=\operatorname{lld}\left(y_{1}\right)=y_{0}$.

Definition 5.1. The top node pairs, $T N \subseteq T \times T^{\prime}$, for a given upper bound $\tau$ are defined as the set of all pairs $(x, y)$ for which the following conditions hold:

(1) There is a node $x^{\prime}, l l d\left(x^{\prime}\right)=l l d(x), \operatorname{post}\left(x^{\prime}\right) \leq \operatorname{post}(x)$, s.t. $\left\|v_{x^{\prime}}-v_{y}\right\|_{1} \leq \tau$ and $\forall_{\left(x^{*}, y^{*}\right)>_{l}\left(x^{\prime}, y\right)}\left(\left\|v_{x^{*}}-v_{y^{*}}\right\|_{1}>\tau\right)$.

(2) There is a node $y^{\prime}, \operatorname{lld}\left(y^{\prime}\right)=l l d(y)$, post $\left(y^{\prime}\right) \leq \operatorname{post}(y)$, s.t. $\left\|v_{x}-v_{y^{\prime}}\right\|_{1} \leq \tau$ and $\forall_{\left(x^{*}, y^{*}\right)>_{l}\left(x, y^{\prime}\right)}\left(\left\|v_{x^{*}}-v_{y^{*}}\right\|_{1}>\tau\right)$.

(3) There is no pair $\left(x^{*}, y^{*}\right)>_{l}(x, y)$ for which (1) and (2) hold.

Example 5.2. The set of top node pairs for the trees in Figure 1, $\tau=2$, is $T N=\left\{\left(x_{2}, y_{3}\right),\left(x_{4}, y_{3}\right),\left(x_{5}, y_{4}\right),\left(x_{6}, y_{6}\right),\left(x_{7}, y_{7}\right)\right\}$. The cells representing top node pairs are marked with green in Figure 3.

Only the distances between top node pairs need to be considered. Top node pairs are compatible with subtree and edits pruning, and guarantee that no subproblem is computed more than once.

Computing top node pairs efficiently. Different from keyroot nodes, top node pairs cannot be computed for each tree in isolation since they depend on the structure of both input trees. Checking the conditions in Definition 5.1 for each node pair $(x, y) \in T \times T^{\prime}$ is quadratic in the number of nodes and does not scale to large trees.

We propose Algorithm 3 to compute top node pairs in linear time. The algorithm iterates over the nodes in a top-down fashion in decreasing order of their postorder IDs (line 4). Only node pairs with neighborhood distance no larger than $\tau$ are considered; to find these pairs, we iterate over all node pairs $(x, y)$ with $\mid \operatorname{post}(x)-$ $\operatorname{post}(y) \mid \leq \tau$. If we have not seen a pair with the same leftmost leaf descendants before (line 5), $(x, y)$ is a new top node pair and is appended to the initially empty list $T N$ (line 9); the index of $(x, y)$ in $T N$ is used to mark the pair of leftmost leaf descendants as visited (line 10). Otherwise, we have a top node pair stored in $T N$ at the index position $S[(\operatorname{lld}(x), \operatorname{lld}(y))]$. We need to verify if $y$ is an ancestor of the previously stored node $\left(\operatorname{post}(y)>\operatorname{post}\left(t_{y}\right)\right)$ and update it (lines 6-7). We do not need to verify $x$ since we iterate over the nodes in $T$ in decreasing postorder.

TopDiff. We present TopDiff (Algorithm 4) that leverages both subtree pruning (gray) and edits pruning (light blue), and at the same time avoids redundant computations using top node pairs 


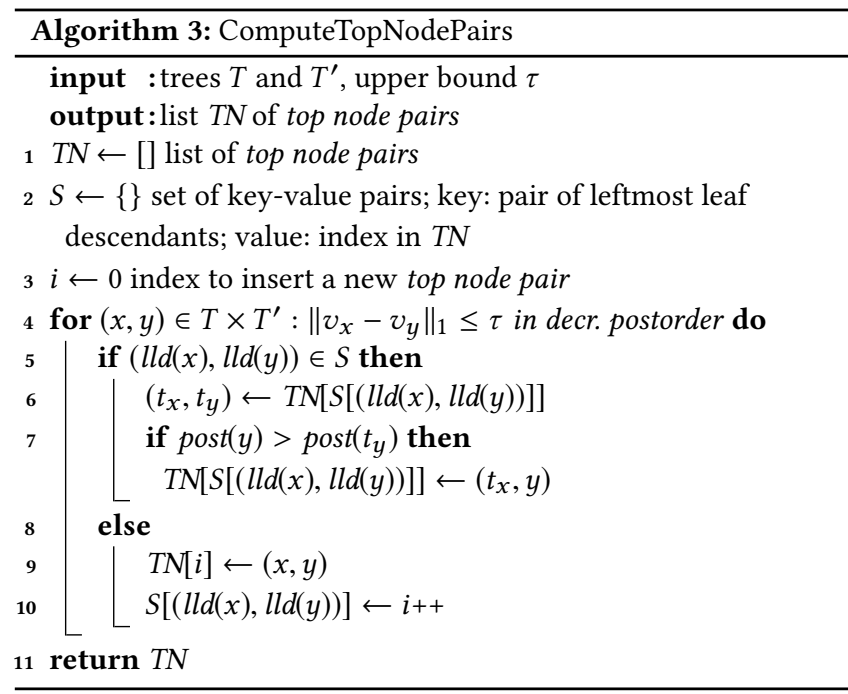

(green). TopDiff uses list $T N$ provided by Algorithm 3 to iterate over all relevant subtree pairs: $T N$ stores the top node pairs in decreasing postorder, and the neighborhood distance of each pair is within $\tau$. In line 8 , the distances between the subtree pairs that are computed as a by-product of the top node pair $(x, y)$ are stored in TD. $(i, j)<_{l}(x, y)$ implies that $T_{i}$ and $T_{j}^{\prime}$ are subtrees of $T_{x}$ and $T_{y}^{\prime}$, respectively.

Theorem 5.3. Correctness. Given two trees, $T$ and $T^{\prime}$, and an upper bound $\tau$, TopDiff computes $\delta_{\tau}\left(T, T^{\prime}\right)$.

Proof. We show that the distance between any subtree pair $\left(T_{x}, T_{y}^{\prime}\right), x \in T, y \in T^{\prime}$, is either correctly computed or the pair is irrelevant (i.e., $\left\|v_{x}-v_{y}\right\|_{1}>\tau$ ). We distinguish two cases for a pair $(x, y)$. (1) $(x, y)$ is a top node pair or there is a top node pair $\left(x_{t}, y_{t}\right)$ such that $\left(x_{t}, y_{t}\right)>_{l}(x, y)$ : TopDiff computes matrix $F D$ for $\left(x_{t}, y_{t}\right)$ following the algorithm of [25], i.e., the distance for the pair $\left(T_{x}, T_{y}^{\prime}\right)$ is correctly computed. Due to the postorder traversal of the top node pairs, the distances between all subtrees required for the node pair $(x, y)$ are already available. (2) Otherwise, the pair $(x, y)$ is irrelevant: Assume $\left\|v_{x}-v_{y}\right\|_{1} \leq \tau$, then according to Def. 5.1 $(x, y)$ is a top node pair or there is a top node pair $\left(x_{t}, y_{t}\right)$ such that $\left(x_{t}, y_{t}\right)>_{l}(x, y)$, which contradicts our assumption.

Complexity analysis. To analyze the complexity of TopDiff we make use of the following lemmas.

Lemma 5.4. The following relationship holds for the sum of the subtree sizes of a tree $T$ :

$$
\sum_{x \in T}\left|T_{x}\right|=\sum_{x \in T} \operatorname{depth}(x) \leq|T| \operatorname{depth}(T)
$$

Proof. Each node $x \in T$ contributes to the subtree size of all its ancestors exactly once, which is $\operatorname{depth}(x)$. The depth of each node $x$ is smaller or equal to the maximum depth in $T$, $\operatorname{depth}(T)$.

Lemma 5.5. Given trees $T, T^{\prime}$, and an upper bound $\tau$, a node $x \in T$ appears in at most $\min \left\{\right.$ leaves $\left.\left(T^{\prime}\right), 2 \tau+1\right\}$ top node pairs:

$$
\left|\left\{\left(x^{\prime}, y^{\prime}\right) \in T N: x^{\prime}=x\right\}\right| \leq \min \left\{\text { leaves }\left(T^{\prime}\right), 2 \tau+1\right\}
$$

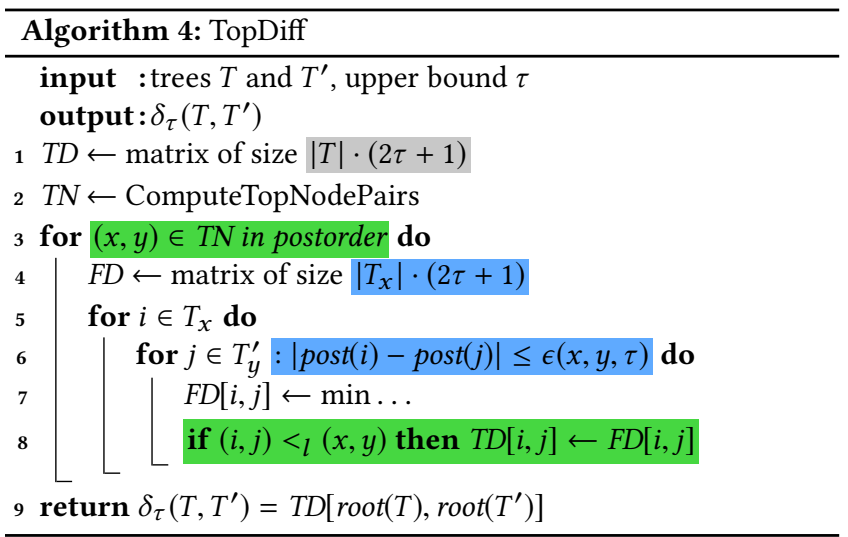

Proof. Leaves. Definition 5.1 implies that there is at most one top node pair for each pair of leaves of $T$ and $T^{\prime}$. Since the left-hand node of each pair is fixed to $x$, there can be at most leaves $\left(T^{\prime}\right)$ such pairs. Tau. All top node pairs $(x, y) \in T N$ satisfy $\left\|v_{x}-v_{y}\right\|_{1} \leq \tau$. The number of pairs for a specific $x$ can be at most $2 \tau+1$ due to the postorder difference: $\left\|v_{x}-v_{y}\right\|_{1} \leq \tau \Rightarrow|\operatorname{post}(x)-\operatorname{post}(y)| \leq \tau$.

Theorem 5.6. TopDiff runs in $O(n d \tau \min \{l, \tau\})$ time and $O(n \tau)$ space for trees with $n$ nodes, depth $d$, upper bound $\tau$, and l leaves.

Proof. Runtime. We count the number of subproblems computed by Algorithm 4 as the number of distance values computed in line 7 . We thereby consider the bound given by edits pruning in line 6 (cf. Section 4).

$$
\sum_{(x, y) \in T N}\left|T_{x}\right|(2 \tau+1)=(2 \tau+1) \sum_{(x, y) \in T N}\left|T_{x}\right|
$$

At most every node $x \in T$ can appear in some top node pair. By Lemma 5.5, there are at most $\min \left\{\right.$ leaves $\left.\left(T^{\prime}\right), 2 \tau+1\right\}$ top node pairs for each $x$. Thus, we can bound the sum as follows.

$$
(2 \tau+1) \sum_{(x, y) \in T N}\left|T_{x}\right| \leq(2 \tau+1) \min \left\{\text { leaves }\left(T^{\prime}\right), 2 \tau+1\right\} \sum_{x \in T}\left|T_{x}\right|
$$

With Lemma 5.4 we bound the sum of the subtree sizes and conclude the final complexity.

$$
\begin{aligned}
& (2 \tau+1) \min \left\{\text { leaves }\left(T^{\prime}\right), 2 \tau+1\right\} \sum_{x \in T}\left|T_{x}\right| \leq \\
& (2 \tau+1) \min \left\{\text { leaves }\left(T^{\prime}\right), 2 \tau+1\right\}|T| \operatorname{depth}(T)=O(n d \tau \min \{l, \tau\})
\end{aligned}
$$

Memory. The space complexity is dominated by the matrices TD and $F D$ that store intermediate results. The size of both matrices is at most $|T| \cdot(2 \tau+1)$, resulting in space complexity $O(n \tau)$.

\section{DEALING WITH DEEP TREES}

TopDiff runs in time $O(n d \tau \min \{l, \tau\})$. For the frequent case of shallow data trees $[4,5]$, TopDiff runs in linear time. If, however, $d=O(n)$, the runtime is $O\left(n^{2} \tau \min \{l, \tau\}\right)$. We discuss how to avoid this worst case using depth-based pruning [25]. Depth-based pruning cannot be used together with top node pairs and introduces redundant computations. It is therefore important to apply depthbased pruning only when the runtime would otherwise be superlinear. 
Depth-based pruning. The depth of node $x$, depth(x), is the number of $x$ 's ancestors. Assume that two node pairs $(x, y)$ and $\left(x^{\prime}, y^{\prime}\right)$, such that $x^{\prime}$ is a descendant of $x$ and $y^{\prime}$ is a descendant of $y$, are in a mapping with $\operatorname{cost} \tau$. Due to edit mapping conditions (cf. Section 3), the ancestors of $x^{\prime}$ can be mapped only to the ancestors of $y^{\prime}$. Hence, the nodes on the paths from $x$ to $x^{\prime}$ and from $y$ to $y^{\prime}$ can be mapped only to each other. Therefore, it must hold that $\operatorname{depth}\left(x^{\prime}\right)-\operatorname{depth}(x) \leq \tau+1$, in particular, $\operatorname{depth}\left(x^{\prime}\right)-\operatorname{depth}(x) \leq$ $\epsilon(x, y, \tau)+1$; similar conditions must hold for $y$ and $y^{\prime}$.

The depth-based pruning is shown in dark blue, line 5 of Algorithm 2 (TouzetDepth). Touzet [25] gives a runtime bound of $O\left(n \tau^{3}\right)$ for this algorithm. We refine the complexity to $O\left(n \tau^{2} \min \{d, \tau\}\right)$, which is better for shallow trees and a high upper bound.

Lemma 6.1. Algorithm 2 runs in $O\left(n \tau^{2} \min \{d, \tau\}\right)$ time.

Proof. Touzet showed that the time complexity of Algorithm 2 is $O\left(n \tau^{3}\right)$ [25]. We now tighten it by introducing tree depth and using the results of Theorem 5.6. Subtree and edits pruning allow us to sum up the number of subproblems as $\sum_{(x, y):\left\|v_{x}-v_{y}\right\| \leq \tau}\left(\left|T_{x}\right|(2 \tau+\right.$ $1))=(2 \tau+1) \sum_{(x, y):\left\|v_{x}-v_{y}\right\| \leq \tau}\left|T_{x}\right| \leq(2 \tau+1)^{2} \sum_{x \in T}\left|T_{x}\right|$. We use Lemma 5.4 to sum up the subtree sizes of $T$. Depth-based pruning limits the depth of each subtree to $\tau$. Then, a node contributes to at most $\min \{\operatorname{depth}(T), \tau\}$ subtrees. Hence, $\sum_{x \in T}\left|T_{x}\right| \leq$ $|T| \min \{\operatorname{depth}(T), \tau\}$. The final bound is $(2 \tau+1)^{2} \sum_{x \in T}\left|T_{x}\right| \leq$ $(2 \tau+1)^{2}|T| \min \{\operatorname{depth}(T), \tau\}$.

Example 6.2. The cells marked with dark blue in Figure 4 correspond to the node pairs that pass the depth-based pruning with $\tau=2$, that is, the pairs $\left(x_{5}, x^{\prime}\right)$ for which $\operatorname{depth}\left(x^{\prime}\right)-\operatorname{depth}\left(x_{5}\right) \leq$ $\epsilon\left(x_{5}, y_{4}, \tau\right)+1$. Nodes $x_{1}$ and $x_{3}$ do not pass the depth-based pruning because $\operatorname{depth}\left(x_{1}\right)=\operatorname{depth}\left(x_{3}\right)=3>\operatorname{depth}\left(x_{5}\right)+\epsilon\left(x_{5}, y_{4}, \tau\right)+1=2$.

TopDiff $^{+}$: Linear runtime for deep trees. The goal is to avoid the quadratic complexity of TopDiff for deep trees with depth $d=O(n)$ without giving up its efficiency for the frequent case of shallow trees. A simple algorithm to achieve this goal uses top node pairs for trees with depth $d=O(\tau)$ and switches to depth-based pruning for deeper trees. However, as we show in our experiments, this approach performs poorly in practice: The true cost does not only depend on the depth but also on the structure of the trees. We propose structure-aware cost functions to identify the break-even point between top node pairs and depth-based pruning.

Our cost functions estimate the number of subproblems that must be computed. The exact number of subproblems depends on both input trees and is too expensive to compute. We propose an approximation that runs in $O(n)$ time.

In our approximation, we focus on the left-hand input tree $T$ and sum up the sizes of all its subtrees that could potentially be considered after the pruning. $\operatorname{cost}$ (TopDiff) is the cost estimate of Algorithm 4 (top node pairs), cost(TouzetDepth, $\tau$ ) estimates the cost of Algorithm 2 (depth-based pruning).

Cost of TouzetDepth. The depth of each subtree is limited by the upper bound value $\tau$ unless the depth is smaller than $\tau$. Let $\left|T_{x}^{\tau}\right|$ be the subtree size of $T_{x}$ where the depth of each node is limited by $\tau$. Using Lemma 5.4, we define the cost of depth-based pruning as

$$
\operatorname{cost}(\text { TouzetDepth, } \tau)=\sum_{x \in T}\left|T_{x}^{\tau}\right|=\sum_{x \in T} \min \{\tau, \operatorname{depth}(x)\} \text {. }
$$

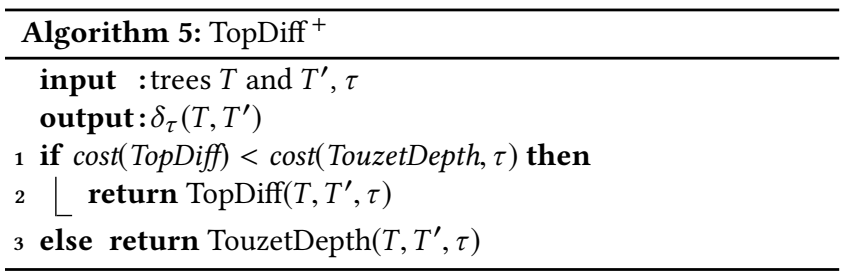

The depth of all nodes can be precomputed in linear time. Thus, the sum can be calculated in a single for-loop over the nodes of $T$.

Cost of TopDiff. Definition 5.1 implies that for each pair of leaf nodes at most one top node pair exists. We sum up the sizes of the largest possible subtrees for each leaf node in $T$. Let $T^{l}$ be the largest subtree of $T$ that has $l$ as the leftmost leaf descendant, that is, $T^{l}=T_{x} \Leftrightarrow \operatorname{lld}(x)=l \wedge \forall_{x^{\prime} \in T, x^{\prime} \neq x}\left(\operatorname{lld}\left(x^{\prime}\right)=l \Rightarrow\left|T_{x^{\prime}}\right|<\left|T_{x}\right|\right)$. Then, we define the cost of TopDiff, independently on $\tau$, as

$$
\operatorname{cost}(\text { TopDiff })=\sum_{x \in \text { leaves }(T)}\left|T^{l}\right| \text {. }
$$

The subtree sizes, leaf nodes, and leftmost leaf descendants can be precomputed in linear time. Then, the sum can be computed in a single for-loop over all leaf nodes of $T$.

TopDiff ${ }^{+}$, Algorithm 5, uses our estimates to execute TopDiff when $\cos t$ (TopDiff) $<\operatorname{cost}$ (TouzetDepth, $\tau$ ), and TouzetDepth otherwise. In Section 8, we experimentally evaluate the cost estimates, show their accuracy, and the break-even points for increasing $\tau$.

\section{TED INDEPENDENT ON THE BOUND}

The TED algorithms TopDiff ${ }^{+}$(Algorithm 4) and TouzetDepth (Algorithm 2) require an upper bound value $\tau$ as input parameter. These algorithms will return the correct edit distance $\delta\left(T, T^{\prime}\right)$ only if $\tau \geq \delta\left(T, T^{\prime}\right)$ is an upper bound on the edit distance. Unfortunately, computing a tight upper bound is expensive (quadratic in the tree size), and a loose upper bound will lead to slow execution times (TopDiff and TouzetDepth are at least quadratic in $\tau$ ).

In this section, we design a TED algorithm that does not require an upper bound $\tau$. We initialize $\tau$ to a lower bound. As proposed by Touzet [25], we double $\tau$ in each round until $\tau$ is an upper bound. Since the $\tau$-based algorithms may return incorrect results if $\tau$ is not an upper bound, a stopping condition that guarantees correctness is required. Touzet does not provide such a condition.

Stopping condition. We devise a stopping condition and build it into our algorithm. Our stopping condition is based on a claim by Hütter et al. [14]. It states when the result of a $\tau$-bounded algorithm, denoted as $\delta_{\tau}\left(T, T^{\prime}\right)$, is good enough. Hütter et al. [14] give only an intuition for the proof; we formally prove the claim in Lemma 7.1. The final stopping condition follows in Theorem 7.2.

LEMMA 7.1. For a given upper bound $\tau$, the following holds:

$$
\delta_{\tau}\left(T, T^{\prime}\right) \leq \tau \Leftrightarrow \delta\left(T, T^{\prime}\right) \leq \tau
$$

Proof. ( $L \Rightarrow R$ ) The cost of a possibly not optimal edit mapping is below the upper bound $\tau$. Thus, certainly the optimal edit mapping is below $\tau$.

$(R \Rightarrow L)$ The proof is by exhaustion. We consider two cases depending on the relationship between the tree edit distance $\delta\left(T, T^{\prime}\right)=$ 


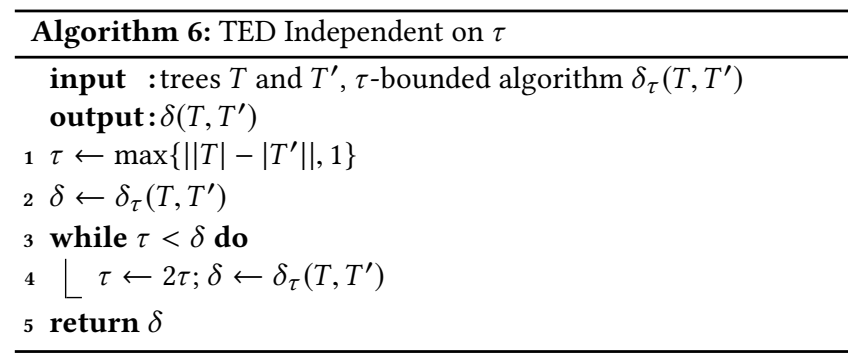

$D$ and the upper bound $\tau$. Assume that the minimum-cost edit mapping between $T$ and $T^{\prime}$ requires $d$ deletions, $i$ insertions, and $r$ renames, i.e., $\delta\left(T, T^{\prime}\right)=D=d+i+r$.

(i) $D \leq \tau$. Then, $d+i+r \leq \tau \Rightarrow d+i \leq \tau$ because $r \geq 0$. Then, the edits pruning allows as many deletions and insertions as in the optimal mapping. Thus, $\delta_{\tau}\left(T, T^{\prime}\right)=\delta\left(T, T^{\prime}\right)=D \leq \tau$.

(ii) $D>\tau$. Then, $d+i+r>\tau$. There are two sub-cases.

(ii 1$) d+i \leq \tau$. Then, as in (i), $\delta_{\tau}\left(T, T^{\prime}\right)=\delta\left(T, T^{\prime}\right)$. But, $\delta\left(T, T^{\prime}\right)=$ $D>\tau$. Thus, $\delta_{\tau}\left(T, T^{\prime}\right)>\tau$.

(ii 2$) d+i>\tau$. There are two possibilities. (a) An edit mapping between $T$ and $T^{\prime}$ with at most $\tau$ deletions and insertions does not exist. In order to find a TED mapping, we need to increase $\tau$. (b) An edit mapping exists but it is not optimal. However, the cost of the optimal edit mapping exceeds $\tau$, i.e., $\delta\left(T, T^{\prime}\right)=D>\tau \Rightarrow$ $\delta_{\tau}\left(T, T^{\prime}, \tau\right)>\tau$.

THEOREM 7.2. Stopping condition:

$$
\delta_{\tau}\left(T, T^{\prime}\right) \leq \tau \Rightarrow \delta_{\tau}\left(T, T^{\prime}\right)=\delta\left(T, T^{\prime}\right)
$$

Proof. Follows from Lemma 7.1.

Final algorithm. Using the stopping condition (Theorem 7.2), we devise Algorithm 6 that computes the exact TED without prior knowledge of an upper bound. The algorithm executes one of the $\tau$-bounded algorithms, e.g., TopDiff ${ }^{+}$or TouzetDepth, iteratively. At first (line 1), $\tau$ is the difference of the input tree sizes which obviously is a lower bound for TED (we use 1 to avoid ||$T|-| T^{\prime}||=$ $0) . \tau$ is doubled at each step (line 4) for as long as the stopping condition is not satisfied (line 3). In each iteration, a $\tau$-bounded algorithm is executed with an increased value of $\tau$ (line 4).

THEOREM 7.3. The time complexity of Algorithm 6 applied with the $\tau$-bounded algorithm TopDiff ${ }^{+}$is $O\left(\log (\delta) n \delta^{3}\right)$, where $\delta=\delta\left(T, T^{\prime}\right)$.

Proof. TopDiff ${ }^{+}$is executed a logarithmic number of times since we double the value of $\tau$ in each iteration round. The largest possible value of $\tau$ is $2 \delta$.

\section{EXPERIMENTS}

We compare our algorithms, TopDiff and TopDiff ${ }^{+}$, with the algorithm by Touzet [25] on real-world and synthetic data. We implemented all algorithms in $\mathrm{C}++$. The experiments are executed on a laptop computer with Intel i5 CPU and 8GB RAM, running Debian 10 OS. The source code and the experimental setup (including data preparation scripts) are available online (http://tree-editdistance.dbresearch.uni-salzburg.at/).
Table 2: Data trees and their sizes

\begin{tabular}{lllrrr}
\hline Source & Tree & Version & \#nodes & depth & \#leaves \\
\hline \multirow{5}{*}{ LinuxRepo } & linux & v5.6-rc1 & 214591 & 13 & 71542 \\
& linux & v5.6-rc2 & 214612 & 13 & 71549 \\
& linux & v5.6-rc3 & 214624 & 13 & 71553 \\
& linux & v5.6-rc4 & 214621 & 13 & 71552 \\
\hline \multirow{5}{*}{ Wikidata } & Q30 & 1156772506 & 122155 & 26 & 40406 \\
& Q30 & 1156772394 & 122103 & 26 & 40391 \\
& Q40 & 1153700198 & 82493 & 26 & 27009 \\
& Q937 & 1151291349 & 82465 & 26 & 27002 \\
& Q937 & 1152908796 & 30641 & 26 & 10178 \\
Synthetic & LB2000 & - & 30613 & 26 & 10171 \\
& FB2000 & - & 2000 & 1000 & 1000 \\
& FB11 & - & 2002 & 1001 & 1001 \\
\hline
\end{tabular}

Table 3: TED and size difference between tree pairs

\begin{tabular}{llllrr}
\hline Pair & Tree & $T$ & $T^{\prime}$ & $\delta\left(T, T^{\prime}\right)$ & $\left\|T|-| T^{\prime}\right\|$ \\
\hline L1 & linux & v5.6-rc4 & v5.6-rc1 & 113 & 30 \\
L2 & linux & v5.6-rc4 & v5.6-rc2 & 55 & 9 \\
L3 & linux & v5.6-rc4 & v5.6-rc3 & 28 & 3 \\
Q30 & Q30 & 1156772506 & 1156772394 & 54 & 52 \\
Q40 & Q40 & 1153700198 & 1151291349 & 30 & 28 \\
Q937 & Q937 & 1152908796 & 1152908745 & 30 & 28 \\
\hline
\end{tabular}

Algorithms. TopDiff is our Algorithm 4 with top node pairs pruning. TopDiff + is our Algorithm 5 that switches between top node pairs and depth-based pruning using our cost estimates. Touzet [25] uses subtree and edits pruning, i.e., Touzet is Algorithm 2 without the colored part in line 5. TouzetDepth is Algorithm 2. AutoStop, Algorithm 6, computes TED independently of the upper bound $\tau$ using one of TopDiff, TopDiff+, Touzet, or TouzetDepth. APTED [23] is the state-of-the-art, $\tau$-independent TED algorithm. Due to its quadratic memory requirements, APTED could be executed only for the small, synthetic datasets.

Datasets. We collected large trees from two different domains. LinuxRepo are directory structures of different versions of the linux kernel repository (https://github.com/torvalds/linux). Wikidata are tree representations of JSON-formatted Wikidata entities (https: //www.wikidata.org/). Synthetic are full binary trees of different shapes as in [22]; LB stands for left-branch tree (each left child is a leaf or has two children, each right child is a leaf), ZZ - zig-zag tree (at each level, alternately either the left or the right child has two children, and the other child is a leaf), FB - perfect binary tree. LB and ZZ are deep trees with depth $d=O(n)$ for trees with $n$ nodes. LB and ZZ represent trees for which Touzet incur a high resp. a low level of redundant computations. We do not consider pairs of so-called right-branch trees (symmetric to LB) since their computation is identical to that of LB pairs when the traversal order is reversed. Table 2 and Table 3 summarize characteristics of the trees and the tree pairs used in our tests, respectively. 
Table 4: Number of subproblems and runtime (in seconds)

\begin{tabular}{llrrrrrr}
\hline Measure & Algorithm & L1 & L2 & L3 & Q30 & Q40 & Q937 \\
\hline \multirow{4}{*}{ \#sp $\times 10^{6}$} & TopDiff & 224 & 145 & 61 & 121 & 43 & 14 \\
& TopDiff+ & 339 & 229 & 106 & 152 & 55 & 18 \\
& Touzet & 468 & 297 & 117 & 1310 & 465 & 161 \\
& TouzetDepth & 468 & 297 & 117 & 1310 & 465 & 161 \\
\hline \multirow{5}{*}{ time(s) } & TopDiff & 11.7 & 7.7 & 3.2 & 4.8 & 1.7 & 0.6 \\
& TopDiff+ & 11.1 & 7.4 & 3.2 & 4.7 & 1.7 & 0.6 \\
& Touzet & 20.8 & 13.2 & 5.3 & 38.7 & 13.9 & 4.6 \\
& TouzetDepth & 24.1 & 15.4 & 6.0 & 45.0 & 15.8 & 5.3 \\
\hline
\end{tabular}

Impact of $\tau$. We evaluated the impact of the upper bound parameter $\tau$ on the performance. The input trees are identical pairs of trees: Linux (linux v5.6-rc4), Q30 (wikidata Q30 version 1156772506), FB, $\mathrm{LB}$, and ZZ. We measured the number of subproblems and the runtime shown in Figures 5a-5e resp. 6a-6e. In most of the cases (Linux, Q30, FB, LB) TopDiff and TopDiff+ are similar and better than TouzetDepth in both the number of subproblems and runtime.

Flat trees (Linux, Q30, FB). For small values of $\tau$ (3 for Linux and FB, 2 for Q30), TouzetDepth computes fewer subproblems than TopDiff. Compared to Touzet, the depth-based pruning of TouzetDepth reduces the number of subproblems only for low values of $\tau$ and quickly converges to Touzet. This happens because $\tau$ becomes higher than the tree depth, and TouzetDepth cannot prune additional subproblems. For $\tau>3$, TopDiff always computes fewer subproblems than the competitors, and is faster for $\tau \geq 2$. Even for high values of $\tau$, on FB trees TopDiff is two orders of magnitude better than APTED regarding subproblems and runtime.

Deep trees $(L B, Z Z)$. For TouzetDepth to have an advantage, the depth of a tree must be significantly larger than $\tau$. The depth of LB and $\mathrm{ZZ}$ trees is 1000 . The tree shape may significantly influence the algorithm's performance. The details of that are described by Pawlik and Augsten [22]. Although the trees are deep, our top node pairs prune significantly more subproblems (around three orders of magnitude) than TouzetDepth for LB trees with similar runtime reductions. For such trees, TopDiff is more than an order of magnitude better than APTED, whereas TouzetDepth degenerates quickly for $\tau>20$. In this case, TouzetDepth redundantly computes many distance values. In the case of ZZ trees, TouzetDepth computes fewer subproblems than TopDiff with a break-even point around $\tau=220$. For the runtime, the break-even point is already at $\tau=150$. TopDiff+ always chooses to use the depth-based pruning. All algorithms experience a break-even point with APTED around $\tau=100$ APTED uses an efficient decomposition strategy that is tailored to $\mathrm{ZZ}$ trees and is more efficient than $\tau$-based pruning for larger values of $\tau$. Note that APTED is not applicable to very large trees due to its quadratic space complexity.

Computing TED independently on $\tau$. For every tree pair listed in Table 3, we execute AutoStop with different pruning algorithms (TopDiff, TopDiff+, Touzet, TouzetDepth). Recall that AutoStop starts with $\tau=|| T|-| T^{\prime}||$, and doubles $\tau$ at each iteration step until the true TED is computed. Table 4 shows number of subproblems and runtime (in seconds) for each of the pruning algorithms.
We make two observations. First, the depth-based pruning improvement of TouzetDepth brings only a negligible reduction of the number of subproblems compared to Touzet. This is caused by the small depth of the trees that offer limited pruning possibilities. The implementation overhead of depth-based pruning has a negative impact on the runtime. Although the number of subproblems of TouzetDepth is always smaller than that of Touzet, the runtime values are always higher. Second, our algorithm, TopDiff, always computes fewer subproblems than the competitors. For Wikidata tree pairs (Q30, Q40, Q937), TopDiff computes at least 10 times fewer subproblems. This leads to runtime reductions of more than $80 \%$. For pairs L1 to L3, we compute $47 \%$ to $52 \%$ fewer subproblems. TopDiff+ shows runtime results similar to TopDiff. Though, the number of subproblems is slightly higher. This is due to the cost estimates that switch to top node pairs pruning later than possible.

Finding a break-even point. We evaluated the accuracy of our cost estimation for TopDiff and TouzetDepth using five pairs of equal trees and varying $\tau$. Figure 7 shows the results. We compared the exact subproblem ratio TopDiff/TouzetDepth (green) with the cost estimation ratio TopDiff/TouzetDepth (blue), i.e., $\operatorname{cost}$ (TopDiff)/ $\operatorname{cost}$ (TouzetDepth, $\tau$ ) (cf. Section 6). The lines would overlap when the estimates are exact. Values below 1 mean that TopDiff should be used because it results in fewer subproblems than TouzetDepth. For Linux and FB trees, our estimates choose TopDiff at $\tau=5$ whereas TopDiff is better than TouzetDepth already at $\tau=4$. For tree Q30, that difference is 3. For LB tree, TopDiff is correctly chosen for all values of $\tau$. This means that even for some deep trees, we can correctly choose TopDiff. The $\mathrm{ZZ}$ tree is an example when the estimates are less accurate.

\section{CONCLUSION}

We considered minimal edit-based diffs between large trees, known as the tree edit distance (TED). Most real-world data consists of flat trees for which no efficient solution existed. The previously proposed algorithm by Touzet [25] suffers from redundant subproblem computations. Moreover, an upper bound value for the tree edit distance must be known upfront. Our TopDiff algorithm is guaranteed to avoid redundant computations and is efficient for large and flat trees. TopDiff ${ }^{+}$detects cases when a special pruning for deeper trees should be applied. We proposed a solution based on TopDiff ${ }^{+}$ that solves TED in $O\left(n \delta^{3} \log \delta\right)$ time, where $n$ is the number of tree nodes and $\delta$ is the size of the minimal diff. We proved structureaware asymptotic bounds of the algorithms. Our experiments on real-world and synthetic data showed that our solution can run orders of magnitude faster than the state of the art.

Acknowledgements. This work was supported by the Austrian Science Fund (FWF): P 29859.

\section{REFERENCES}

[1] Tatsuya Akutsu. 2010. Tree edit distance problems algorithms and applications to bioinformatics. IEICE Trans. on Information and Systems E93-D, 2 (2010), 208-218.

[2] Kiyoko F. Aoki, Atsuko Yamaguchi, Yasushi Okuno, Tatsuya Akutsu, Nobuhisa Ueda, Minoru Kanehisa, and Hiroshi Mamitsuka. 2003. Efficient tree-matching methods for accurate carbohydrate database queries. Genome Informatics 14 (2003), 134-143.

[3] Nikolaus Augsten, Denilson Barbosa, Michael Böhlen, and Themis Palpanas. 2011. Efficient top-k approximate subtree matching in small memory. IEEE Trans. on Knowledge and Data Engineering 23, 8 (2011), 1123-1137. 


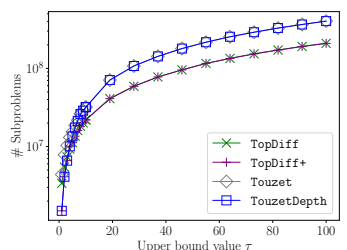

(a) linux

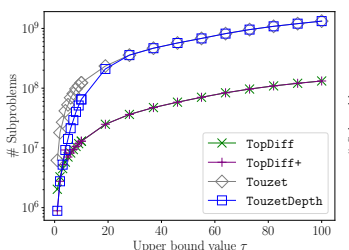

(b) Q30

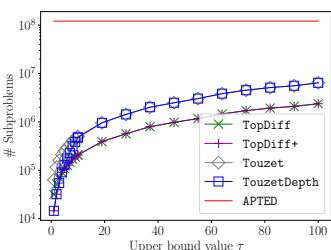

(c) FB

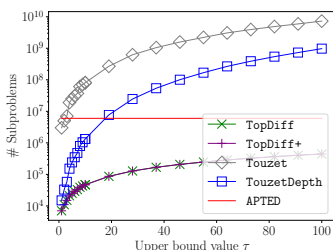

(d) LB

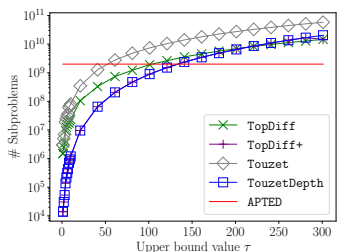

(e) $\mathrm{ZZ}$

Figure 5: Number of subproblems for varying $\tau$ and different inputs (Y-axis in log scale).

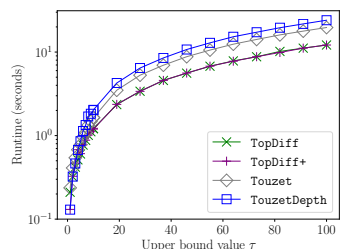

(a) linux

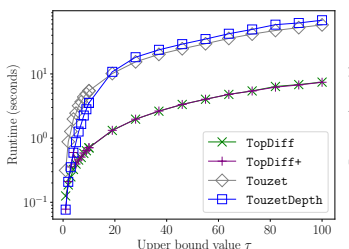

(b) Q30

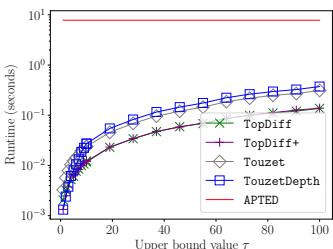

(c) FB

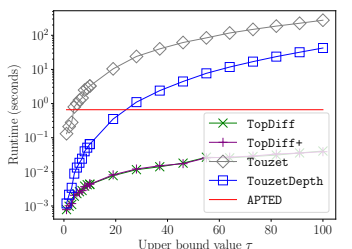

(d) LB

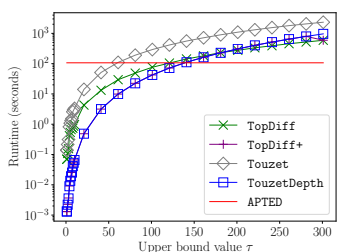

(e) $\mathrm{ZZ}$

Figure 6: Runtime (in seconds) for varying $\tau$ and different inputs (Y-axis in log scale).

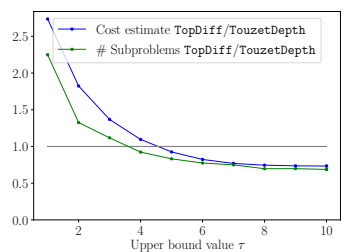

(a) linux

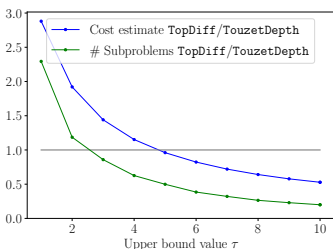

(b) Q30

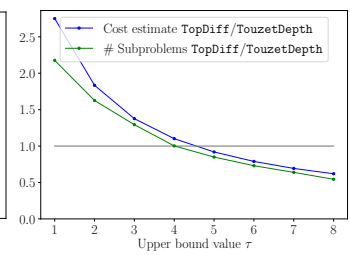

(c) FB

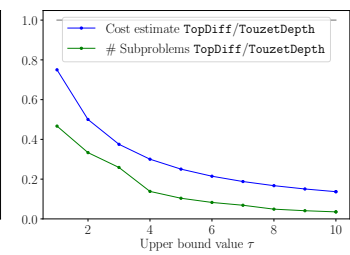

(d) LB

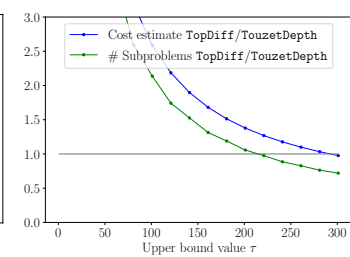

(e) $\mathrm{ZZ}$

Figure 7: Accuracy of our cost estimates in TopDiff+.

[4] Mohamed Amine Baazizi, Dario Colazzo, Giorgio Ghelli, and Carlo Sartiani. 2019 Parametric schema inference for massive JSON datasets. VLDB fournal 28, 4 (2019), 497-521.

[5] Denilson Barbosa, Laurent Mignet, and Pierangelo Veltri. 2005. Studying the XML web: gathering statistics from an XML sample. World Wide Web 8, 4 (2005), 413-438.

[6] John Bellando and Ravi Kothari. 1999. Region-based modeling and tree edit distance as a basis for gesture recognition. In Int. Conf. on Image Analysis and Processing. 698-703.

[7] Mahdi Boroujeni, Mohammad Ghodsi, MohammadTaghi Hajiaghayi, and Saeed Seddighin. 2019. $1+\epsilon$ approximation of tree edit distance in quadratic time. In ACM SIGACT Symposium on Theory of Computing. 709-720.

[8] Karl Bringmann, Pawel Gawrychowski, Shay Mozes, and Oren Weimann. 2018 Tree edit distance cannot be computed in strongly subcubic time (unless APSP can). In ACM-SIAM Symposium on Discrete Algorithms. 1190-1206.

[9] Davi de Castro Reis, Paulo Braz Golgher, Altigran Soares da Silva, and Alberto H. F. Laender. 2004. Automatic web news extraction using tree edit distance. In WWW. 502-511.

[10] Erik D. Demaine, Shay Mozes, Benjamin Rossman, and Oren Weimann. 2009. An optimal decomposition algorithm for tree edit distance. ACM Trans. on Algorithms 6, 1 (2009).

[11] Jan Finis, Robert Brunel, Alfons Kemper, Thomas Neumann, Norman May, and Franz Färber. 2015. Indexing highly dynamic hierarchical data. Proceedings of the VLDB Endowment 8, 10 (2015), 986-997.

[12] Amaury Habrard, José Manuel Iñesta, David Rizo, and Marc Sebban. 2008. Melody recognition with learned edit distances. In Structural, Syntactic, and Statistical Pattern Recognition: Joint IAPR Int. Workshop, SSPR \& SPR. 86-96.

[13] Holger Heumann and Gabriel Wittum. 2009. The tree-edit-distance, a measure for quantifying neuronal morphology. Neuroinformatics 7, 3 (2009), 179-190.

[14] Thomas Hütter, Mateusz Pawlik, Robert Loschinger, and Nikolaus Augsten. 2019 Effective Filters and Linear Time Verification for Tree Similarity Joins. In IEEE Int. Conf. on Data Engineering.

[15] Shahab Kamali and Frank Wm Tompa. 2013. Retrieving Documents with Mathematical Content. In Int. Conf. on Research and Development in Information Retrieval. 353-362.
[16] Tomohiro Kan, Shoichi Higuchi, and Kouichi Hirata. 2014. Segmental mapping and distance for rooted labeled ordered trees. Fundam. Inform. 132, 4 (2014), 461-483.

[17] Yeonjung Kim, Jeahyun Park, Taehwan Kim, and Joongmin Choi. 2007. Web information extraction by HTML tree edit distance matching. In Int. Conf. on Convergence Information Technology. 2455-2460.

[18] Philip N. Klein, Srikanta Tirthapura, Daniel Sharvit, and Benjamin B. Kimia. 2000. A tree-edit-distance algorithm for comparing simple, closed shapes. In ACM-SIAM Symposium on Discrete Algorithms. 696-704.

[19] Zhiwei Lin, Hui Wang, and Sally I. McClean. 2010. Measuring tree similarity for natural language processing based information retrieval. In Int. Conf. on Applications of Natural Language to Information Systems. 13-23.

[20] Bin Ma, Lusheng Wang, and Kaizhong Zhang. 2002. Computing similarity between RNA structures. Theoretical Computer Science 276, 1-2 (2002), 111-132.

[21] Mateusz Pawlik and Nikolaus Augsten. 2011. RTED: a robust algorithm for the tree edit distance. Proceedings of the VLDB Endowment 5, 4 (2011), 334-345.

[22] Mateusz Pawlik and Nikolaus Augsten. 2015. Efficient computation of the tree edit distance. ACM Trans. on Database Systems 40, 1 (2015).

[23] Mateusz Pawlik and Nikolaus Augsten. 2016. Tree edit distance: robust and memory-efficient. Information Systems 56 (2016), 157-173.

[24] Huo-Chung Tai. 1979. The tree-to-tree correction problem. f. ACM 26, 3 (1979), 422-433.

[25] Hélène Touzet. 2007. Comparing similar ordered trees in linear-time. Journal of Discrete Algorithms 5, 4 (2007), 696-705.

[26] Lusheng Wang and Kaizhong Zhang. 2008. Space efficient algorithms for ordered tree comparison. Algorithmica 51, 3 (2008), 283-297.

[27] Yuan Wang, David J. DeWitt, and Jin-Yi Cai. 2003. X-Diff an effective change detection algorithm for XML documents. In IEEE Int. Conf. on Data Engineering.

[28] Kaizhong Zhang. 1995. Algorithms for the constrained editing distance between ordered labeled trees and related problems. Pattern Recognit. 28, 3 (1995), 463-474.

[29] Kaizhong Zhang and Dennis Shasha. 1989. Simple fast algorithms for the editing distance between trees and related problems. SIAM f. Comput. 18, 6 (1989), $1245-1262$.

[30] Kaizhong Zhang, Rick Statman, and Dennis Shasha. 1992. On the editing distance between unordered labeled trees. Inform. Process. Lett. 42, 3 (1992), 133-139. 